\title{
Análisis de las actividades propuestas en dos programas de estudio chilenos en el eje de Estadística y Probabilidad
}

\author{
Analysis of the proposed activities in two Chilean study programs \\ in the statistics and probability axis
}

Nicolás Andrés Sánchez Acevedo

Blanca Rosa Ruiz Hernández

\begin{abstract}
RESUMEN
En este trabajo se analizan las actividades propuestas en los programas de estudio de $7^{\circ}$ y $8^{\circ}$ básico del eje de Estadística de Chile. Se hace uso de una de las propuestas que ha mostrado utilidad para fomentar la comprensión estadística, que es la inferencia informal. Esta busca una articulación entre la generalización y la incertidumbre al hacer juicios basándose en muestras para fomentar el aprendizaje estadístico de manera holística. La metodología seguida fue cualitativa bajo una técnica de análisis de contenido. Los resultados muestran que una gran cantidad de actividades propuestas en los programas de estudio presentan una marcada tendencia de lenguaje centrado en la muestra. En algunos casos, las actividades sugieren la posibilidad de hacer interpretaciones conjuntas al integrar conceptos de medidas de centro, posición, variabilidad de la muestra, apoyado por la conexión con el tipo de contexto que presentan las actividades. En el caso particular de los programas de $8^{\circ}$ básico (antiguo y vigente) no se encontró evidencia de estas componentes, viéndose restringida la posibilidad para desarrollar el razonamiento inferencial, no obstante lo anterior, se encontraron algunas actividades que pueden ser potencialmente usadas como base por el profesor para integrar las componentes de inferencia informal y contexto y desarrollar este tipo de razonamiento.
\end{abstract}

Palabras clave: estadística y probabilidad, programas de estudio, cualitativo, aprendizajes esperados, inferencia informal.

\section{Abstract}

This work analyzes the activities proposed in the 7th and 8th grade study programs of the Statistics axis of Chile. One of the proposals that has been proven useful in promoting statistical understanding, which is informal inference, is used. This seeks an articulation between generalization and uncertainty when making judgments based on samples to promote statistical learning in a holistic way. The methodology followed was qualitative under a content analysis technique. The results show that a large number of activities proposed in the study programs have a marked tendency for sample-centered language. In some cases, activities suggest the possibility of making joint interpretations by integrating concepts of measures of center, position, variability of the sample, supported by the connection with the type of context that the activities present. In the particular case of the 8th grade programs (old and current), no evidence of these components was found, the possibility to develop inferential reasoning was restricted, however, some activities were found that can potentially be used as base by the teacher to integrate the components of informal inference and context and develop this type of reasoning.

Keywords: statistics and probability, study programs, qualitative, expected learning, informal inference. 


\section{INTRODUCCIÓN}

La investigación a nivel curricular en el área de estadística y probabilidad ha sido protagonista de varios debates académicos a partir de la creciente incorporación de este eje en diversos países (NCTM, 2000; GAISE, 2016). Una de las áreas que ha cobrado mayor relevancia es el análisis del currículo de matemáticas en el eje de Probabilidad y Estadística. Han sido puestos a discusión y análisis los objetivos que proponen, el tipo de actividades y la coherencia entre ambos (objetivos y actividades) con las directrices internacionales en educación estadística (NCTM, 2000; GAISE, 2016).

El estudio de la estadística proporciona las herramientas e ideas necesarias para enfrentarse de manera adecuada a distintos tipos de información, por lo que se hace necesario que en la actualidad todo ciudadano adquiera la capacidad de interpretar y comprender información que proviene de diferentes medios y fenómenos cotidianos (Batanero, 2002). En este sentido, la inferencia es la herramienta principal de la estadística, pues permite leer, entender e interpretar de manera objetiva las conclusiones derivadas de los análisis de datos a partir de una muestra. Esto implica razonar de forma juiciosa con base en la muestra, es decir, visualizar los datos como un agregado que permite mirar a la población (Konold, Higgins, Russell y Khalil, 2015). Los juicios que se realicen sobre la representatividad de una muestra llevan a tomar la decisión sobre si el uso de una inferencia es o no confiable (Aridor y Ben-Zvi, 2018).

Tanto el contexto como el razonamiento estadístico son componentes esenciales para desarrollar la comprensión de grandes ideas estadísticas (Doerr, delMas y Makar, 2017). Tanto el contexto como la inferencia informal son dependientes, pues el contexto provee de riqueza a las afirmaciones que se hacen desde una muestra a una población y son parte del sustento para la formulación de inferencias que cobren validez en ese contexto (Makar, Bakker y Ben-Zvi, 2011). El razonamiento

\footnotetext{
Nicolás Andrés Sánchez Acevedo. Profesor-investigador en la Escuela de Matemática y Estadística de la Universidad Central de Chile. Actualmente está finalizando estudios de doctorado en Ciencias con mención en Matemática Educativa por el Instituto Politécnico Nacional, México. Es miembro de la Red Latinoamericana en Educación Estadística (RELIEE), del Grupo de Investigación en Didáctica de las Matemáticas (SIDM), España, y del Comité Latinoamericano de Matemática Educativa (CLAME). Las líneas de trabajo que desarrolla son análisis de libros de texto, desarrollo profesional docente, didáctica de la estadística y razonamiento algebraico en estudiantes de secundaria. Correo electrónico: nicolas1983@cicata.edu.mx. ID: https:// orcid.org/0000-0002-0665-6102.

Blanca Rosa Ruiz Hernández. Profesora-investigadora del Tecnológico de Monterrey, NL, México. Trabaja en el área de Estadística y Educación Estadística. Es doctora en Didáctica de la Matemática por la Universidad de Granada, España. Es miembro fundador de la Red Latinoamericana en Educación Estadística (RELIEE) y la Red de Investigación e Innovación en Educación Estadística y Matemática Educativa (RIIEEME). Entre sus investigaciones más importantes se encuentra "Una propuesta de diálogo entre la investigación y la docencia: Seminario Repensar las matemáticas” (2015) y otras en didáctica de la estadística como "La relación entre la variable aleatoria y la variable estadística". Correo electrónico: bruiz@tec.mx. ID: https:// orcid.org/0000-0003-0157-3866.
} 
estadístico permite describir el proceso mediante el cual los estudiantes razonan con ideas estadísticas como centralización, variabilidad, posición, gráficas y tablas y hacen conexiones entre conceptos (Ben-Zvi y Garfield, 2004; Garfield y Ben-Zvi, 2008).

Una de las propuestas que ha mostrado ser útil para integrar el contexto, los conceptos y procedimientos estadísticos vinculados con la estadística descriptiva, es la inferencial informal, que permite un proceso razonado para establecer generalizaciones con base en la evidencia de los datos de una muestra (Gal, 2019; Makar y Rubin, 2009). Esta propuesta emerge como "respuesta a las dificultades que presentan estudiantes en el uso adecuado de procesos estadísticos" (Erickson, 2006, p. 1). La relevancia está en la posibilidad de dar sustento a afirmaciones o conclusiones sin el apoyo de herramientas estadísticas formales, pero sí con características relevantes de los datos al momento de inferir (lenguaje basado en la incertidumbre, sustento en datos, generalización). Por lo tanto, permite la integración de los conceptos estadísticos por medio del desarrollo de juicios, predicciones, la integración de conocimientos previos, la articulación de evidencia a través de argumentos (Zieffler, Garfield, delMas y Reading, 2008), la inclusión de la noción de incertidumbre, dar peso al valor de agregado en una muestra y evidencia sobre los datos (Makar y Rubin, 2009).

Con la intención de atender las actuales demandas que la sociedad necesita (GAISE, 2016; MEC, 2007; Mineduc, 2015; NCTM, 2000), varios países han tenido que reconfigurar los currículos de educación escolar. La Guía para la Evaluación y Enseñanza de la Educación Estadística (GAISE, de sus siglas en inglés) explicita los siguientes objetivos en esta área: (I) enseñar el pensamiento estadístico, (II) centrarse en la comprensión conceptual, (III) integrar datos reales con un contexto y un objetivo definido, (IV) fomentar el aprendizaje activo, (V) hacer uso de tecnología para explorar conceptos y analizar datos, y (VI) usar las evaluaciones para mejorar y evaluar el aprendizaje de los estudiantes. Estos seis objetivos son promovidos con la inferencia informal, pero los objetivos II y III son los más coherentes con un desarrollo de razonamiento inferencial informal.

Chile no ha estado ajeno a este proceso. Incorporó en su currículum objetivos que buscan el desarrollo del pensamiento estadístico. En reemplazo del antiguo documento de los Objetivos fundamentales y contenidos mínimos obligatorios de la Educación Básica y Media (Mineduc, 2009) se proponen las vigentes bases curriculares (Mineduc, 2015). Así también los programas de estudio sufrieron modificaciones, incorporándose mayor cantidad de actividades sugeridas al profesor; se incluyó el uso de tecnologías bajo cuatro habilidades (modelar, argumentar y comunicar, resolver problemas y representar) como piedra angular del currículo chileno.

El currículum en Chile se compone de cuatro documentos oficiales: las bases curriculares, los programas de estudio, los planes de estudio y los manuales escolares, descritos en la tabla 1 según su prioridad. 
Tabla 1. Documentos oficiales que integran el currículum en Chile.

\begin{tabular}{|c|c|}
\hline Documento oficial & Descripción \\
\hline Bases curriculares & $\begin{array}{l}\text { Indican los aprendizajes comunes para los alumnos y alumnas durante su trayectoria escolar } \\
\text { (Mineduc, 2015, p. 23). Se muestran los lineamientos generales para todas las asignaturas que componen } \\
\text { la educación básica y media en Chile }\end{array}$ \\
\hline Programas de estudio & $\begin{array}{l}\text { Organizan temporalmente los objetivos de aprendizaje. Constituyen una propuesta que organiza en } \\
\text { el tiempo los objetivos de aprendizaje para facilitar a los/las docentes su quehacer en el aula } \\
\text { (Mineduc, } 2015 \text {, p. } 23 \text { ). Incluyen los objetivos de aprendizaje por unidad, proponen actividades sugeridas } \\
\text { al profesor para el diseño de la enseñanza, junto a la(s) habilidad(es) en cada tema }\end{array}$ \\
\hline Planes de estudio & $\begin{array}{l}\text { Muestran la organización del tiempo escolar y establecen el tiempo mínimo que se estima necesario } \\
\text { destinar a cada una de las asignaturas para cumplir (MinEDUC, 2015, p. 23). Son una base sugerida para } \\
\text { la distribución de las horas lectivas que se debe distribuir en cada unidad y cada tema específico de la } \\
\text { unidad de aprendizaje }\end{array}$ \\
\hline Libros de textos & $\begin{array}{l}\text { Son la concreción de los objetivos propuestos en las bases curriculares, por medio de actividades para } \\
\text { los estudiantes. Estos se estructuran en cuatro unidades (o ejes) temáticos: Álgebra y Funciones, } \\
\text { Geometría, Estadística y Probabilidad y Números }\end{array}$ \\
\hline
\end{tabular}

Fuente: Elaboración personal.

Los objetivos de la base curricular vigente ( $7^{\circ}$ básico a $2^{\circ}$ medio) en la unidad de Estadística y Probabilidad es que "todos y todas las estudiantes aprendan a realizar análisis, inferencias y obtengan información a partir de datos estadísticos (...) de forma crítica, utilizando la información para validar sus opiniones y decisiones" (MinEdUC, 2015, p. 100), lo cual es coherente con lo planteado por GAISE (2016) y NCTM (2000) en sus objetivos orientadores para la enseñanza de la estadística.

Los documentos que integran el currículo en los distintos niveles educativos son un soporte fundamental para los profesores debido al poder que les otorgan en la planificación de su enseñanza (Brown, 2009). Sin una adecuada profundización y pertinencia en el uso de los documentos curriculares, cualquier tipo de ajuste será en vano y descontextualizado, dado que es necesario que estos se adapten a las nuevas necesidades para mejorar la enseñanza (Davis y Krajcik, 2005).

La unidad de Estadística y Probabilidad es una de las más demandantes debido al auge que ha tenido esta área en el currículo en muchos países (Del Pino y Estrella, 2012). Es por lo anterior que las actividades propuestas en los programas de estudio son un aspecto central para el trabajo del profesor, pues sugieren lineamientos orientadores para la enseñanza. La relación entre el profesor y los materiales curriculares brinda oportunidades de acción para un cambio pedagógico en la enseñanza y el aprendizaje (Remillard, 2000; Remillard y Bryans, 2004).

Si bien es cierto que la evidencia se ha centrado mayormente en el análisis de libros de texto, la profundización en el programa de estudio en el área de estadística ha sido escasa, por lo que analizar programas de estudio de diferentes periodos 
curriculares es relevante para conocer si se han incorporado propuestas coherentes con las actuales directrices internacionales. En este escrito se pretende responder a la pregunta " ¿Las actividades propuestas en los programas de estudio vigentes de $7^{\circ} \mathrm{y}$ $8^{\circ}$ básico de Chile en el eje de Estadística y Probabilidad incorporan las sugerencias para desarrollar el razonamiento inferencial estadístico con respecto a los antiguos programas de estudio?" Esta pregunta de investigación se traduce en el siguiente objetivo: analizar las actividades propuestas en los programas de estudio vigentes de $7^{\circ}$ y $8^{\circ}$ básico de Chile en la unidad de Estadística y Probabilidad y compararlas con los antiguos programas de estudio y relacionarlos con los aprendizajes esperados que las sustentan.

\section{Antecedentes}

Variadas investigaciones sobre inferencia informal tienen su justificación en las dificultades encontradas en el tratamiento de la inferencia formal. Por ejemplo, algunas dan cuenta de la ausencia de experiencias con eventos estocásticos, que son la base de la inferencia estadística (Pfannkuch, 2005). Para García-Ríos y Sánchez (2015) esta exclusión de experiencias con eventos estocásticos ha llevado a enseñar estadística bajo la idea del adiestramiento, es decir, profundización en los cálculos, técnicas para graficar y reglas mecánicas aisladas, carentes de significado y de un contexto de los datos.

Según Zieffler et al. (2008) las dificultades que tienen los estudiantes en el aprendizaje de la inferencia estadística giran en torno a la falta de adaptación a la lógica del pensamiento estadístico, la intolerancia a la ambigüedad, la falta de habilidad para reconocer la estructura de un problema de inferencia, la comprensión incompleta que tienen de conceptos fundamentales involucrados, como la distribución, variación, muestreo y distribuciones muestrales.

La necesidad de suplir los vacíos en el aprendizaje de la estadística ha llevado a realizar mayores esfuerzos en materia de investigación, específicamente en esta área curricular, dada la importancia atribuida y los cambios que se han realizado en distintos países (Pérez, Ruiz y Hugues, 2019). No obstante, algunos resultados siguen evidenciando inconsistencias entre los objetivos propuestos para la enseñanza de la estadística y las actividades sugeridas al profesor para el diseño de la enseñanza, tanto en programas de estudio como en libros de texto (Díaz-Levicoy, Batanero, Arteaga y Gea, 2015; Morales y Ruiz, 2013; Vásquez y Alsina, 2017).

En México, López-Mojica (2013) realizó un análisis de actividades propuestas en programas de estudio centradas en contexto probabilista, evidenciándose que, en el ciclo preescolar, estos documentos parecieran no considerar relevante el desarrollo de este tipo de pensamiento, pues no identifica lecciones introductorias para conducir hacia este tipo de pensamiento. 
El trabajo llevado a cabo por Maldonado y Ojeda (2009) sobre análisis de planes y programas se extendió a la propuesta institucional en primaria en el eje de análisis de información, encontrándose una disminución en los temas de enseñanza de la estadística, focalizándose solo en el uso de tablas, gráficas, pictogramas y diagramas como medios para organizar y comparar datos.

En este mismo contexto, López-Mojica, Ojeda y Salcedo (2018) analizaron los programas de estudio de seis grados escolares de primaria y las actividades en los libros de texto de primero y segundo grados de primaria en el tema de Estadística y Probabilidad. Entre las conclusiones más relevantes se evidencia una desconexión entre los planes y programas y los libros de texto (gratuitos) en el tema de probabilidad. Esta desconexión se presenta, por una parte, porque los programas de estudio analizados no incluyen temas relacionados con el tratamiento de la probabilidad, reduciendo los objetivos planteados al tratamiento de la información y al pensamiento determinista, sin incluir la posibilidad de inducir los fenómenos aleatorios. Ciertas lecciones sobre la idea de incertidumbre, con base en actividades alrededor de fenómenos aleatorios, aluden a situaciones con dados, pero su presentación en libros de texto y programas de estudio se limita al énfasis de "culto al número" (López-Mojica, Ojeda y Salcedo, 2018, p. 100), es decir, actividades que promueven un sentido mecánico de la estadística.

Batanero, Gea, Arteaga y Contreras (2014) llevaron un estudio sobre la metodología y contenidos en las directrices curriculares españolas en la educación primaria y secundaria obligatoria. El foco de este trabajo fue analizar formas de enseñanza y contenidos. Encontraron resultados alentadores, ya que las orientaciones curriculares sobre estadística dan cuenta de las recomendaciones que hacen diversos autores, como Franklin, Kader, Mewborn, Moreno, Peck, Perry y Scheaffer (2005) y Watson (2006), concluyendo que las orientaciones españolas promueven el razonamiento a partir de datos empíricos, la necesidad de estos en el análisis de fenómenos naturales, la comprensión de la omnipresencia de la variación, la importancia del contexto en estadística así como de percibir, cuantificar y explicar la variación. Esta investigación (Batanero et al., 2014) hace un análisis sobre los objetivos presentes en los directrices curriculares, pero no tiene como objetivo analizar la conexión entre los objetivos y las actividades propuestas en las mismas directrices o en los libros de texto.

En Chile el trabajo en esta área ha sido escaso, sin embargo Morales y Ruiz (2013) realizan una comparación de la pertinencia de los objetivos que proponen los currículos de Chile y España, evidenciándose que la estructura y los objetivos en ambos currículos es coherente y gradual, sugiriendo el desarrollo de competencias o habilidades para que los estudiantes registren, clasifiquen, lean e interpreten datos de diversas fuentes de información. Entre las diferencias encontradas está que los objetivos del currículo español promueven la recopilación de información por medio de encuestas, lo que no pasa en el currículo chileno. En cambio, en el currículo 
chileno, para el tema de comparación de distribuciones hay un fuerte énfasis en el uso de diagrama de puntos y de tallo y hojas.

Trabajos como los de Morales y Ruiz (2013) y Batanero et al. (2014) dan cuanta de una coherencia entre los objetivos declarados en estos documentos y propuestas como GAISE (2016) y NCTM (2000), pero dejan un vacío sobre la vinculación entre objetivos y actividades, planteándose la necesidad de seguir explorando sobre la pertinencia de los objetivos curriculares. En Chile, explorar la coherencia entre los objetivos de aprendizaje y las actividades sugeridas al profesor para el diseño de la enseñanza proporciona la oportunidad de observar si se han llevado a cabo esos cambios y cómo se han realizado.

\section{Marco Conceptual}

El marco conceptual de este trabajo se estructura en tres apartados: (1) la descripción de los objetivos de aprendizaje de los programas de estudio de $7^{\circ}$ básico (Mineduc, 2000, 2016a) y $8^{\circ}$ básico (Mineduc, 2002, 2016b); (2) el marco de razonamiento de inferencia informal propuesto por Makar y Rubin (2009) a través de sus componentes, las cuales permiten analizar las actividades propuestas en los programas de estudio, y (3) la propuesta de contexto estadístico enunciada por Pfannkuch (2011).

\section{La estadística en los programas de estudio}

En la tabla 2 se describen, de forma general, los objetivos específicos de los programas de estudio de $7^{\circ}$ y $8^{\circ}$ básico en el eje de Estadística y Probabilidad vigentes (Mineduc, 2016a, 2016b) y los anteriores (Mineduc, 2000, 2002).

Los programas de estudio antiguos de $7^{\circ}$ básico (Mineduc, 2000) y $8^{\circ}$ básico (Mineduc, 2002) no tenían incorporada una unidad específica de estadística y probabilidad, siendo algunos temas presentados en otras unidades (ver tabla 2). Los antiguos programas se dividían en números, variaciones proporcionales y números y ecuaciones. Actualmente, los programas vigentes (Mineduc, 2016a, 2016b) cuentan con una unidad específica de Estadística y Probabilidad.

Así mismo, en los objetivos propuestos en los programas de $7^{\circ}$ básico (MinEDuc, 2000) y $8^{\circ}$ básico (Mineduc, 2002) no había referencia a la posibilidad de hacer inferencias, pues el foco de estos era descriptivo y con contextos superficiales. En el caso de los programas vigentes de $7^{\circ}$ y $8^{\circ}$ básico (Mineduc, 2016a, 2016b) los objetivos incluyen como habilidad realizar inferencias. Por ejemplo, en el programa de $7^{\circ}$ básico se plantea la posibilidad de estimar características de la población a partir de un muestreo y en $8^{\circ}$ básico se plantea la comprensión de medidas de tendencia central y de posición, lo que implica conocer el contexto de los datos para interpretar estas medidas de resumen sobre las características poblacionales. 
Tabla 2. Comparación de los objetivos en el eje de Estadística y Probabilidad en los programas de $7^{\circ}$ y $8^{\circ}$ básico chileno.

\begin{tabular}{|c|c|}
\hline \multicolumn{2}{|c|}{ Programa de estudio de $7^{\circ}$ básico } \\
\hline Objetivos del programa (Mineduc, 2000) & Objetivos del programa (Mineduc, 2016a) \\
\hline $\begin{array}{l}\text { - Análisis de información utilizando como indicador de } \\
\text { dispersión el recorrido de la variable, y como medidas de } \\
\text { tendencia central la moda, la media y la mediana } \\
\text { - Presentación de información en tablas. Análisis de } \\
\text { información } \\
\text { - Presentación de información en tablas de frecuencias } \\
\text { relativas y construcción de gráficos circulares }\end{array}$ & $\begin{array}{l}\text { - Estimar el porcentaje de algunas características de una } \\
\text { población desconocida por medio del muestreo } \\
\text { - Representar datos obtenidos en una muestra mediante } \\
\text { tablas de frecuencias absolutas y relativas, utilizando gráficos } \\
\text { apropiados, de manera manual y/o con software educativo } \\
\text { - Mostrar qué comprenden las medidas de tendencia central } \\
\text { y el rango }\end{array}$ \\
\hline \multicolumn{2}{|c|}{ Programa de estudio de $8^{\circ}$ básico } \\
\hline Objetivos del programa (Mineduc, 2002) & Objetivos del programa (MinEDuc, 2016a) \\
\hline $\begin{array}{l}\text { - Análisis de tablas y gráficos estadísticos habitualmente } \\
\text { utilizados en la prensa, asociado con relaciones y } \\
\text { variaciones proporcionales y porcentajes } \\
\text { - Lectura y análisis de encuestas de opinión en relación con } \\
\text { proporciones y porcentajes. Tratamiento de información } \\
\text { - Análisis de tablas y gráficos estadísticos habitualmente } \\
\text { utilizados en la prensa }\end{array}$ & $\begin{array}{l}\text { - Mostrar qué comprenden las medidas de posición, } \\
\text { percentiles y cuartiles } \\
\text { - Evaluar la forma en que los datos están presentados }\end{array}$ \\
\hline
\end{tabular}

Fuente: Elaboración personal.

\section{La inferencia estadística informal}

La inferencia informal ha mostrado ser potencialmente eficaz para desarrollar una comprensión más profunda de los estudiantes en el aprendizaje estadístico. Para Makar y Rubin (2009) la inferencia informal es un enfoque de aprendizaje de la estadística, en el que se prioriza la integración de las herramientas estadísticas, como las medidas de centralización, dispersión, las gráficas y los procedimientos en el centro de la enseñanza, articulando los distintos enfoques en un proceso continuo. Se centra no solo en ver lo que muestran los datos, sino también en brindar procedimientos que permitan ver más allá de estos, es decir, con la intención de desarrollar un pensamiento y razonamiento inferencial apoyados por datos.

El marco propuesto por Makar y Rubin (2009) consta de tres componentes interrelacionadas, las cuales describen de manera esencial la inferencia estadística informal (figura 1).

- Generalizar o predecir al observar más allá de los datos,

- El uso de los datos como evidencia, y

- Empleo de un lenguaje probabilístico al describir generalizaciones, incluyendo ideas informales con cierto grado de certeza a partir de las conclusiones propuestas. 


\section{Inferencia Estadística}

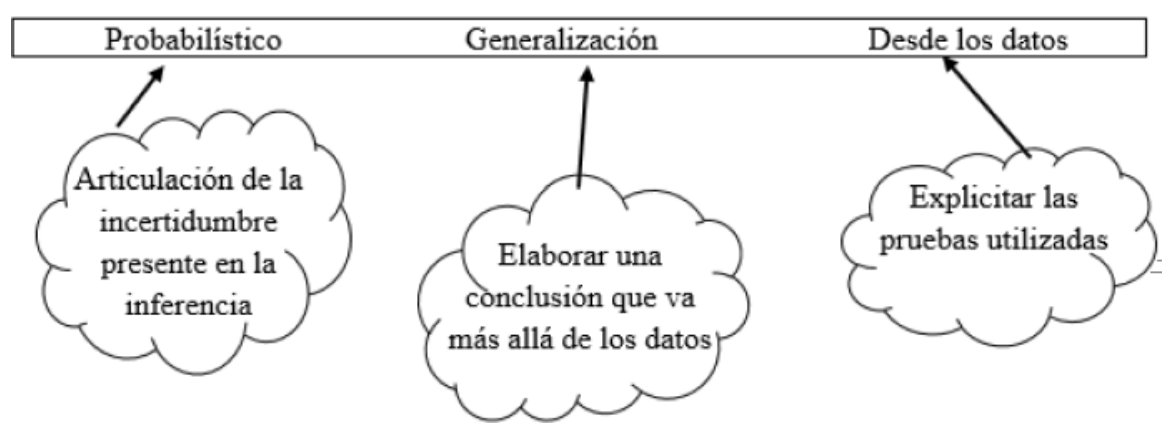

Figura 1. Marco para el pensamiento acerca de la inferencia estadística informal. Fuente: Makar y Rubin, 2009, p. 85.

La integración de las componentes descritas permite evaluar el razonamiento de inferencia informal en estudiantes por medio de:

- Conclusiones que se extienden más allá de los datos (generalización). Incluyendo predicciones, estimación de parámetros y conclusiones que se deben extender más allá de la descripción de los datos (hacia la población).

- Uso de datos como evidencia, que sostienen esa generalización, predicción o conclusión. Es decir, dar argumentos implícitos o explícitos que justifiquen la generalización y que sea aceptable dentro del contexto real.

- Uso de un lenguaje probabilístico o que denote incertidumbre al relacionar o escribir una generalización, predicción o conclusión. Se refiere a la utilización de verbos que indiquen incertidumbre e incluye la fuerza de la evidencia, indicando, por ejemplo, una estimación subjetiva de la probabilidad basándose en el análisis de los datos (muy probable, poco probable, tal vez, etc.). Indica una comprensión de que las conclusiones obtenidas usando la muestra pueden no ser las de la población debido a la aleatoriedad en el muestreo.

\section{El contexto en estadística}

La idea de contexto parece ser una idea simple de interpretar, específicamente considerando que cada persona y ciudadano tiene un contexto de vida. Pero al contrario de lo que sucede en la vida cotidiana, en el aula de clases el contexto dista mucho de ser una noción simple de abordar (Gal, 2019).

Gal (2019) menciona que para nutrir de riqueza las inferencias, el contexto de los datos: (I) debe ser auténtico, debe ser producido de manera natural, y no artificial ni ficticio, y (II) debe llamar a una verdadera necesidad de saber, la cual debe ser de interés para un ciudadano externo (como políticos, agentes ministeriales, directores de instituciones educativas, profesores a nivel curso, etc.). 
La investigación destaca que el contexto para analizar datos se relaciona directamente con el ciclo investigativo propuesto por Wild y Pfannkuch (1999), compuesto por cuatro dimensiones (el ciclo investigativo, tipos fundamentales de pensamiento estadístico, el ciclo interrogativo y disposiciones). La dimensión II (tipos fundamentales de pensamiento estadístico) alude específicamente a que una parte importante en el desarrollo del pensamiento estadístico es la integración de la estadística con el contexto para hacer inferencias. Para Pfannkuch y Wild (2004) la capacidad de integrar información estadística y de contexto es un elemento fundamental del pensamiento estadístico. Ellos plantean que la información estadística sobre el mundo real está contenida en los resúmenes estadísticos, por lo tanto es imprescindible que funcione la síntesis del conocimiento estadístico sobre ese contexto para extraer lo que se pueda aprender de esos datos.

Para Pfannkuch (2011) el contexto de los datos es un factor que no puede desligarse del aprendizaje estadístico y del razonamiento que permite su desarrollo. Aún cuando Pfannkuch (2011) no define de una manera específica el contexto en estadística, sí distingue entre el uso que se da al contexto al resolver problemas estadísticos y el contexto en ambientes educativos. Con base en esto, considera dos tipos de contexto escolar que afectan el desarrollo del razonamiento inferencial informal: el contexto basado en datos y el contexto basado en experiencias de aprendizaje.

En síntesis, el marco de análisis que se propone en este estudio destaca que las componentes de inferencia informal deben integrarse al analizar una muestra y son el soporte fundamental para elaborar inferencias sobre un fenómeno de estudio y las conclusiones que de él se extraigan en los niveles básicos. De forma paralela, el contexto de los datos brinda un espacio para enriquecer las inferencias, considerando el origen de los datos, el conocimiento del mundo desde donde emergen los datos y las características que las definen.

\section{Metodología}

En este trabajo se adopta un enfoque cualitativo con carácter exploratorio-descriptivo (Pérez-Serrano, 1994). Es de interés comprender y profundizar un fenómeno específico de forma detallada. La técnica de investigación es el análisis de contenido (Krippendorff, 1997).

El objetivo de esta investigación no pretende la generalización, sino profundizar en cómo las actividades propuestas en estos programas orientan la integración de conceptos estadísticos y de contexto para desarrollar el razonamiento inferencial.

Si bien es cierto que los conceptos estadísticos se incluyen de forma gradual a lo largo del currículum de Matemáticas, en particular en la unidad de Estadística y Probabilidad, es en $5^{\circ}$ básico (estudiantes entre 10 y 11 años) que los objetivos de aprendizaje plantean la lectura e interpretación de tablas y gráficos y su relación con 
el contexto. Desde $7^{\circ}$ básico (estudiantes entre 12 y 13 años) y $8^{\circ}$ básico (estudiantes entre 13 y 14 años) se comienza a incrementar la presencia de las ideas y conceptos estadísticos propuestos en los programas de estudio, evidenciándose un énfasis en lo procedimental, complementado con lo conceptual. Especialmente es relevante el análisis y comparación de los programas de estudio de $7^{\circ}$ y $8^{\circ}$ básico (Mineduc, 2000, 2002, 2016a, 2016b), pues estos dos niveles educativos anteceden la educación media obligatoria.

Se consideró para el análisis una muestra intencionada de cuatro programas de estudio de educación básicos, dos programas de $7^{\circ}$ básico (Mineduc, 2000, 2016a) y dos programas de $8^{\circ}$ básico (Mineduc, 2002, 2016ba). Los programas en estos niveles fueron seleccionados por ser de amplio uso por profesores para el diseño de la enseñanza y por ser dos niveles que anteceden la educación media.

Para llevar a cabo el análisis de contenido se siguieron los pasos propuestos por Cobo (2003), quien sugiere:

- Identificar y seleccionar las partes de los libros de texto que presenten tareas relacionadas con estadística y probabilidad.

- Las secciones se transforman en unidades de registro, porciones mínimas de contenido que se analizan por separado.

- Se establecen las unidades de análisis y categorías a considerar para codificar la información. En este trabajo se establecen componentes de inferencia informal y categorías de contexto.

- Se codifican las actividades en la unidad de Estadística y Probabilidad (tratamiento de la información) de los programas de estudio de acuerdo con los indicadores definidos.

- Selección de actividades estadísticas según componentes y categorías de análisis definidas.

\section{Categorías y componentes de análisis}

Se consideraron dos unidades de análisis: inferencia informal y contexto, las cuales describimos de acuerdo a sus componentes.

\section{Inferencia informal.}

La unidad de análisis de inferencia informal está integrada por cuatro componentes. Las tres primeras (generalización, datos como evidencia y uso de leguaje probabilístico) son las componentes propuestas por Makar y Rubin (2009), y como tales se pueden presentar integradas entre sí. La cuarta categoría (lenguaje centrado en la muestra) se propuso por los autores de este trabajo con la finalidad de clasificar y describir aquellas actividades que no presentaran componentes de inferencia informal, pues, 
aunque explícitamente no favorecen el desarrollo de RII, potencialmente sí podrían. Las componentes son:

a) Más allá de los datos (generalización): se analiza si las actividades planteadas incluyen la posibilidad de hacer predicciones, estimaciones de parámetros y/o conclusiones con la idea de hacer descripciones que vayan más allá de los datos.

b) Uso de datos como evidencia: se analiza si las actividades proveen elementos para hacer uso de datos y sustentar generalizaciones en ellos y no en otros aspectos, como por ejemplo las propias creencias.

c) Uso de lenguaje probabilístico para hacer generalizaciones: se analiza si la actividad propuesta permite la posibilidad de plantear respuestas que no sean cerradas (deterministas) y que estén expresadas de manera aleatoria (por ejemplo, se espera que, aproximadamente, con una posibilidad de, es posible, etc.).

d) Uso de lenguaje centrado en la muestra: se analiza si la redacción de las actividades no favorece elaborar inferencias a la población aún cuando el análisis se centra en una muestra. Estas actividades son de carácter descriptivo centrado en la muestra.

\section{Contexto escolar.}

En términos del uso escolar se distinguen dos tipos de contexto: por una parte aquellos usados en la resolución de problemas estadísticos reales, y por otra la forma como se aprecia el contexto para fines curriculares y de profundización de conceptos y procedimientos. Estos dos tipos de contexto se deben tener en cuenta en el aprendizaje estadístico, pues inciden en el desarrollo del RII.

Se describen las dos componentes de contexto utilizadas en esta investigación para el análisis de las actividades de los programas de estudio:

a) Contexto basado en datos: que son aquellos que consideran al contexto real desde el cual surgió el problema (dimensión I del ciclo investigativo de Wild y Pfannkuch, 1999) y se relacionan directamente con que el estudiante resuelva el problema o profundice más en la situación real. Además se requiere que se conozca de manera contextualizada la situación del mundo real.

b) Contexto basado en experiencia de aprendizaje: son aquellas en que los estudiantes resuelven una tarea específica sin los aspectos del contexto real. En este caso, estas tareas tienen el sentido de incluir aspectos para construir aprendizajes, demanda de conocimientos estadísticos previos e inclusión de nuevas ideas o conceptos (Pfannkuch, 2011). 


\section{Discusión de los RESUltados}

El análisis se divide en dos partes: en primer lugar se ejemplifican y describen actividades que muestren alguna de las categorías presentadas y posibles conexiones entre los componentes de la inferencia informal de acuerdo con Makar y Rubin (2009), y en segundo lugar se presentan los resultados de acuerdo con las categorías de inferencia informal y de contexto escolar.

La presencia de las componentes de inferencia informal en las actividades se hizo buscando evidencias, tanto en los enunciados como en las consignas, que permitieran el desarrollo del razonamiento inferencial bajo la integración del uso de datos como evidencia para sustentar afirmaciones por medio de un lenguaje probabilista para ir más allá de la muestra. Esta interacción entre las componentes se apoya en el contexto de los datos desde donde los datos dan significado a las conclusiones.

\section{Tipos de actividades propuestas en los programas de estudio}

\section{De inferencia informal.}

En esta dimensión se busca que los enunciados de las actividades de los programas de estudio sugieran la posibilidad de incluir alguna o todas las componentes que posibiliten el desarrollo de inferencias informales de forma integrada.

\section{Más allá de los datos (posibilidad de generalizar).}

En este componte se busca que el enunciado de la actividad, a partir de una muestra, permita hacer una generalización a partir de los datos disponibles, estimando alguna característica de la población. La figura 2 del programa de estudio vigente de $7^{\circ}$ básico (Mineduc, 2016a) permite ejemplificar la posibilidad de ir más allá de los datos en su enunciado como en sus preguntas.

Participan en el siguiente juego de dados:

Lanzan un dado, si sale el 6, ganan un punto. ¿Qué parte del curso cree usted que ganará un punto? (Expresan en porcentajes, si es necesario).

a. Compare el resultado obtenido de manera experimental con lo que usted creía que saldría como resultado.

b. Si cada estudiante del curso vuelve a lanzar, ¿se obtendrá el mismo resultado?

c. Conjeture lo que ocurre si se pudiera lanzar muchas veces, compare con lo que estiman sus compañeros.

d. ¿Cuántas veces cree que sale el 6, si tira seis veces el dado? (Repetir esto por lo menos 5 veces).

Figura 2. Actividad que permite ir más allá de datos (posibilidad de generalizar) del programa de $7^{\circ}$ básico vigente.

Fuente: Mineduc, 2016a, p. 166. 
En esta actividad de juego de dados la posibilidad de realizar una inferencia emerge del mismo enunciado cuando se pregunta "¿Qué parte del curso cree usted que ganará un punto?” Esta pregunta permite hacer una estimación sobre la proporción de estudiantes que ganarán dicho punto, es decir, cada estudiante plantea un valor para dicha característica poblacional. Sobre esta conjetura, la consigna siguiente pide que el estudiante compare su estimación experimental con lo que de forma teoría se creía que podía suceder. La necesidad de la experimentación para probar una conjetura permite promover el uso de un lenguaje probabilista (restringido al trabajo del profesor). Para las consignas b, c y d se pide una réplica del experimento, fomentando la comprensión de que los resultados están sujetos a la variabilidad de los datos de la muestra y por lo tanto se abre la posibilidad de error al aceptar o rechazar la conjetura basándose en una muestra.

La posibilidad de inferir hace emerger el uso de datos como evidencia para poder generalizar (en este caso, la proporción de estudiantes que ganarán un punto), pues la actividad permite realizar réplicas varias veces, haciendo plausible generar inferencias.

\section{Uso de datos como evidencia.}

La presencia de esta componente en las actividades busca que la atención esté centrada en el uso de datos para validar inferencias y no en la descripción de la muestra. Un ejemplo de actividad que presenta esta componente es tomado del programa de estudio de $7^{\circ}$ básico vigente (Mineduc, 2016a) que se muestra en la figura 3.

Cristián pertenece a la selección del atletismo de su colegio y entrena en lanzamiento de bala para el próximo campeonato entre los colegios de la región.

Consiguió los siguientes resultados en sus lanzamientos:

\begin{tabular}{|c|c|c|c|c|c|c|c|c|}
\hline $\begin{array}{c}\mathrm{N}^{\bullet} \mathrm{DE} \\
\text { LANZAMIENTO }\end{array}$ & 1 & 2 & 3 & 4 & 5 & 6 & 7 & 8 \\
\hline $\begin{array}{c}\text { DISTANCIA } \\
\text { (M) }\end{array}$ & 8,11 & 8,26 & 7,91 & 2,05 & 8,07 & 7,88 & 8,03 & 7,94 \\
\hline
\end{tabular}

, Calculan la media de los resultados.

, En el campeonato de los colegios, Cristián hará 3 lanzamientos. ¿Se puede esperar que los resultados serán similares al promedio de los Lanzamientos del entrenamiento? Explican y comunican la respuesta.

, Determinan la mediana de los resultados y conjeturan sobre los resultados que podría lograr Cristián en el campeonato.

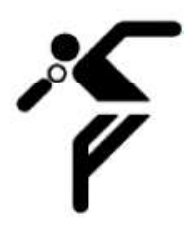

Figura 3. Actividad que permite hacer uso de los datos como evidencia del programa vigente de $7^{\circ}$ básico. Fuente: Mineduc, 2016a, p. 167.

Del enunciado se desprende que los lanzamientos son una muestra de todos los lanzamientos posibles que puede hacer Cristián en la competición. El uso de datos como evidencia se ve reflejada en la primera consigna. El cálculo de la media arit- 
mética sirve como evidencia para predecir (estimar) y responder lo que se pregunta en la segunda consigna, donde el valor de la media aritmética sirve de evidencia para sustentar la inferencia en relación a los lanzamientos de Cristián. La tabla de datos y la secuencia presentada permiten también sustentar inferencia sobre la mediana (consigna tres) para conjeturar los resultados del estudiante en el campeonato. La pregunta " ¿Se puede esperar que los resultados sean similares al promedio de los lanzamientos del entrenamiento?” contempla una comparación entre la información que proporciona la media con la que proporciona la mediana, abriendo la pregunta sobre cuál de las dos medidas podría ser mejor predictor del comportamiento de los lanzamientos de Cristián. El hecho de mostrar esta actividad categorizada en uso de datos como evidencia no excluye las otras dos. El uso de estos datos como evidencia, en este caso permite ir más allá de los datos, en las frases "conjeture" y "¿`se puede esperar...?".

\section{Lenguaje probabilístico.}

La inclusión de esta componente en las actividades se manifiesta cuando tanto el enunciado como las consignas presentan algún tipo de palabra o frase que dé cuenta de la aleatoriedad de los datos a partir del muestreo, es decir, que no sea reflejo de una afirmación determinista. Se busca que el estudiante se percate de que el muestreo introduce la incertidumbre de que la conclusión obtenida a partir de los datos de una muestra puede ser errónea debido al azar. Se debe poder distinguir la incertidumbre de la variabilidad de los datos. La primera es incorporada por el muestreo, la segunda existiría aún si se realizara un censo. La primera da cuenta de la posibilidad de que la conclusión esté equivocada, la segunda se manifiesta en la dificultad de que el estadís-

Realizan la siguiente actividad:

Se miden todos los alumnos de la clase y completan la siguiente tabla:

\begin{tabular}{|l|l|l|l|l|}
\hline Alumno & Estatura & $\begin{array}{l}\text { Frecuencia } \\
\text { absoluta: Cantidad } \\
\text { de veces que } \\
\text { aparece en el } \\
\text { curso }\end{array}$ & Porcentaje & $\begin{array}{l}\text { Frecuencia relativa: } \\
\text { Cantidad de veces que } \\
\text { aparece en el curso/ } \\
\text { cantidad de estudiantes } \\
\text { del curso }\end{array}$ \\
\hline
\end{tabular}

- Elaboran un gráfico de barras.

- Hacen una tómbola y se preguntan cuál es la probabilidad de que el niño elegido tenga una determinada estatura. (Eligen primero las más frecuentes).

, Igual que la pregunta anterior, eligen la menos frecuente.

- Se hacen grupos de niños con estaturas similares y se esconde a los ganadores de la tómbola. Determinan subdivisiones de los grupos para encontrar a los ganadores.

Figura 4. Actividad que hace uso de un lenguaje probabilista del programa vigente de $7^{\circ}$ básico. Fuente: Mineduc, 2016a, p. 169. 
tico adopte exactamente el valor del parámetro. Dado que ninguna de las actividades propuestas en los programas de estudio hace uso de lenguaje probabilista, se muestra en la figura 4 un ejemplo ajustado para describir esta componente en las actividades.

En este caso, la actividad favorece la inclusión del contexto del curso al medir las estaturas de los compañeros del curso. La recopilación de todas las estaturas da la oportunidad de trabajar con los datos de una población y analizar su distribución. La posibilidad de probar una conjetura emergería de la consigna cuatro al tratar de que el estudiante prediga la estatura del ganador en la tómbola. Esta conjetura será más certera si el estudiante elige un intervalo (alguno de los grupos construidos) que un valor puntual. Esta reflexión y el uso de lenguaje probabilístico estaría presente al preguntar “¿cuál es la probabilidad de que tu conjetura sea certera?”. Como se conoce la distribución de la población, el cálculo de la probabilidad será directo. Las conjeturas de los estudiantes se pueden vincular con hipótesis a probar y el resultado final (la estatura del niño seleccionado) con la información que un dato aportaría sobre la población si esta no se conociera. Se esperaría que las conjeturas de los niños y la estatura del niño seleccionado en la tómbola estuvieran en los rangos de mayores datos. Se podría discutir la información que proporciona la distribución sobre el dato seleccionado al azar, pero también sobre la información que el dato seleccionado al azar podría proporcionar sobre las estaturas del curso si no se conociera su distribución. Si la estatura del niño seleccionado en la tómbola no está en los rangos de mayor probabilidad, se daría pie a la discusión de la posibilidad de error debido a la aleatoriedad del muestreo. Esta actividad se podría hacer aún más rica si posteriormente se elige al azar a un niño de otro curso del mismo grado y se pide que, basándose en su estatura, se indique si las estaturas de los niños del otro curso están en el rango de estaturas más frecuentes de su propio curso con una probabilidad alta, baja o media.

\section{Lenguaje centrado en la muestra.}

El uso de lenguaje centrado en la muestra es una componente que permite describir (clasificar) actividades en las que no se evidencia ninguna de las tres componentes anteriores a pesar de hacer uso de una muestra. La figura 5 es una actividad tomada del programa de estudio de $7^{\circ}$ básico antiguo (Mineduc, 2000) que plantea la búsqueda de información que tiene por objetivo la construcción de gráficas y uso de porcentajes, pero no amplía su uso a la elaboración de inferencias. Es de esperarse que la información incluida en los diarios sea una muestra (en la mayoría de los casos es asî), sin embargo, la actividad no aprovecha esta situación para cuestionar, indagar, preguntarse sobre posibles conclusiones de la información que muestra la revista y la validez de las afirmaciones. 
a) Recopilan en diarios o revistas informaciones presentadas en gráficos, incluidos los gráficos circulares.

- Leen y analizan las informaciones: discuten por qué algunas se presentan en gráficos de barras y otras en graficos circulares.

- Relacionan los porcentajes senalados en los gráficos circulares con la porción del área de la circunferencia (aproximadamente) que ocupan. Por ejemplo, si es el $50 \%$ corresponde a la mitad, etc.

Figura 5. Actividad con lenguaje centrado en la muestra del programa de $7^{\circ}$ básico antiguo. Fuente: Mineduc, 2000, p. 119.

\section{Contexto escolar}

Se ha declarado que el aprendizaje de la estadística no puede estar desprovisto del contexto de los datos. Pfannkuch (2011) sugiere proponer actividades en un contexto de aprendizaje que permitan generar estructuras conceptuales para desarrollar el razonamiento inferencial informal, sin embargo debe haber un equilibrio entre los tipos de contextos presentados (basado en datos y basado en experiencias de aprendizaje).

\section{Contexto basado en datos.}

Este tipo de contexto busca que las actividades propuestas en los programas de estudio aporten información relativa al entorno de una investigación de tipo estadística, objetivos, plan de trabajo, fuentes y recolección de datos, análisis, etc. Con esto se espera que las conclusiones hacia la población contengan los elementos que permiten una contextualización casi real del fenómeno bajo estudio para dar mayor validez a la inferencia. Ejemplos de actividades de contexto en datos se muestran en las figuras 6 y 7 .

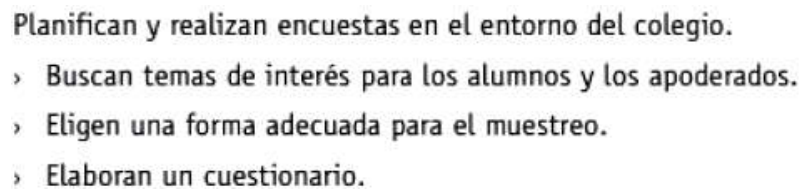

Figura 6. Actividad que presenta contexto de datos del programa de $7^{\circ}$ básico vigente . Fuente: Mineduc, 2016a, p. 166.

La propuesta de actividad de la figura 6 es una actividad extraída del programa de $7^{\circ}$ básico vigente (Mineduc, 2016a) que permite conocer el contexto de los estudiantes dentro del colegio. En esta actividad el contexto estará condicionado por el interés de cada estudiante en la aplicación de la encuesta. La forma en la selección de la muestra de compañeros y las preguntas que se incluyan en el cuestionario proporcionan algunos elementos necesarios para sustentar posibles afirmaciones basadas en la muestra. 
Trabajan en parejas o en grupos: leen informaciones como las que se entregan a continuacion, las comentan y responden a las preguntas que se formulen.

Hasta el año 1999, se habla registrado que en el mundo habla 350 millones de personas vivas infectadas con Hepatitis $B$.

Fuentes: INE, ministerios, servicios publicos y publicaciones periodicas, en general.

Figura 7. Actividad que presenta un contexto de datos del programa de $7^{\circ}$ básico antiguo. Fuente: Mineduc, 200, p. 100.

En el caso de la actividad de la figura 7, si bien es cierto que el contexto viene dado por el enunciado de la actividad, se presenta la fuente desde donde es extraída la información relativa para conjeturar una conclusión. En la actividad se proporciona una fuente desde donde se puede profundizar en la situación real.

\section{Contexto de experiencia de aprendizaje.}

En este caso, el objetivo de las actividades es la profundización en aspectos conceptuales y procedimentales. Este tipo de actividades está desprovisto de los elementos que sustenta el contexto basado en datos, sin embargo, la situación puede presentar un contexto ficticio. Un ejemplo de este tipo de contexto se muestra en la figura 8.

Comparan dos muestras representadas por un gráfico de doble barra que se refiere al agua caída en dos regiones. Los números en el eje horizontal indican los meses (de enero a diciembre) y los del eje vertical indican la cantidad de agua en $\mathrm{mm}$ por $\mathrm{m}^{2}$.

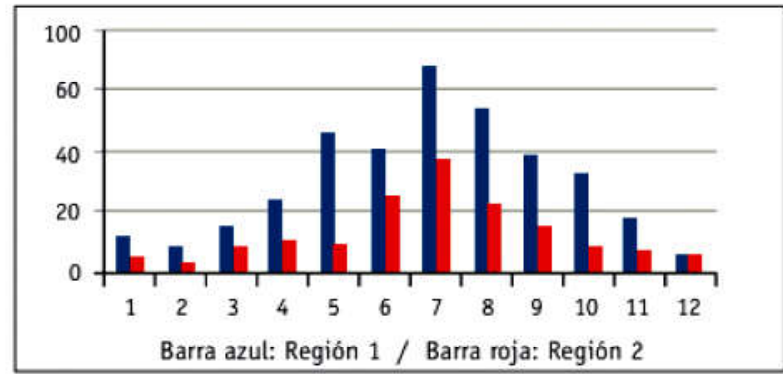

- El gráfico de doble barra, ¿compara de buena forma las coherencias y las diferencias del agua caída? Explican la respuesta.

, Dibujan en el gráfico de barras un gráfico de línea correspondiente.

Figura 8. Actividad que presenta un contexto de experiencia de aprendizaje del programa de $8^{\circ}$ básico vigente. Fuente: Mineduc, 2016b, p. 178.

La actividad de la figura 8 muestra un gráfico de barras para comparar el agua caída en dos regiones distintas. Tanto el enunciado como las consignas no presentan elementos sobre los objetivos del estudio, la fuente de los datos, la toma de la muestra 
o el análisis de datos, país o región, que son aspectos necesarios para poder sustentar y dar validez a una inferencia. Otros aspectos que se evidencian en esta actividad son que en la consigna comparar la precipitación en dos regiones y dibujar un gráfico alternativo, que enfatiza lo procedimental por sobre lo conceptual, se da por asumido que el estudiante sabe diferenciar los tipos de variables que son determinantes en la construcción de gráficas adecuadas y la noción de muestra está equivocada puesto que no es posible que la muestra esté compuesta por un dato para cada mes para cada región.

\section{Categorización de las actividades y posibles integraciones}

En la tabla 3 se muestran las frecuencias de actividades y sus posibles integraciones. De acuerdo con Makar y Rubin (2009), una inferencia informal debe estar integrada por las tres componentes, sin embargo, es posible que las actividades propuestas no presenten dicha integración. Esto significa que el desarrollo de una inferencia se debe basar en los datos de una muestra para poder generalizar haciendo uso de un lenguaje que denote incertidumbre (Vásquez, 2018). En algunos casos tanto el enunciado como las consignas dan cuenta de la presencia de una de las componentes sin integración con las restantes.

Tabla 3. Frecuencia de las actividades para las componentes de Inferencia Informal y contexto en los programas de estudio de $7^{\circ}$ y $8^{\circ}$ básico antiguos y vigentes.

\begin{tabular}{|c|c|c|c|c|}
\hline \multirow[b]{2}{*}{ Componentes } & \multicolumn{4}{|c|}{ Programas de estudio } \\
\hline & $7^{\circ}$ antiguo & $7^{\circ}$ vigente & $8^{\circ}$ antiguo & $8^{\circ}$ vigente \\
\hline \multicolumn{5}{|l|}{ 1. Inferencia informal } \\
\hline $\begin{array}{l}\text { 1.1. Conexión entre el uso de datos como evidencia y posibilidad de ir } \\
\text { más allá de los datos }\end{array}$ & $1(25 \%)$ & $4(26,7 \%)$ & $1(50 \%)$ & $0(0 \%)$ \\
\hline $\begin{array}{l}\text { 1.2. Conexión entre el uso de datos como evidencia y uso del lenguaje } \\
\text { probabilístico }\end{array}$ & $0(0 \%)$ & $0(0 \%)$ & $0(0 \%)$ & $0(0 \%)$ \\
\hline $\begin{array}{l}\text { 1.3. Conexión entre posibilidad de ir más allá de los datos usando un } \\
\text { lenguaje probabilístico }\end{array}$ & $0(0 \%)$ & $0(0 \%)$ & $0(0 \%)$ & $0(0 \%)$ \\
\hline $\begin{array}{l}\text { 1.4. Conexión entre la posibilidad de ir más allá de los datos, con uso de } \\
\text { datos como evidencia por medio de un lenguaje probabilístico }\end{array}$ & $0(0 \%)$ & $0(0 \%)$ & $0(0 \%)$ & $0(0 \%)$ \\
\hline 1.5. Lenguaje centrado en la muestra & $2(50 \%)$ & $9(60 \%)$ & $1(50 \%)$ & $9(69,2 \%)$ \\
\hline \multicolumn{5}{|l|}{ 1.6. Actividades no sugieren conexiones entre los componentes de } \\
\hline 1.7. Descripción de los datos a nivel poblacional & $0(0 \%)$ & $2(13,3 \%)$ & $0(0 \%)$ & $4(30,8 \%)$ \\
\hline \multicolumn{5}{|l|}{ 2. Contexto escolar } \\
\hline 2.1. Contexto basado en datos & $2(50 \%)$ & $4(25 \%)$ & $1(50 \%)$ & $0(0 \%)$ \\
\hline 2.2. Contexto basado en experiencias de aprendizaje & $2(50 \%)$ & $12(75 \%)$ & $1(50 \%)$ & $13(100 \%)$ \\
\hline
\end{tabular}


La tabla 3 muestra un incremento en la cantidad de actividades propuestas en los programas de estudio vigentes (Mineduc, 2016a; 2016b) con relación a los programas antiguos (Mineduc, 2000; 2002). Los programas antiguos no contemplaban una unidad específica para la unidad de Estadística y Probabilidad, aspecto que se incorporó en los programas vigentes de $7^{\circ}$ y $8^{\circ}$ básico. Los programas de $7^{\circ}$ básico (Mineduc, 2000, 2016a), presentan cinco actividades (26,3\% del total de estos dos programas) que sugieren la integración de dos de las tres componentes de inferencia informal (conexión entre el uso de datos como evidencia y posibilidad de ir más allá de los datos). En el caso de los programas de $8^{\circ}$ básico (Mineduc, 2002; 2016b) solo una actividad presenta conexión de estas dos componentes de inferencia informal, sin hacer uso de lenguaje probabilista (6,7\% del total de actividades). La categoría de lenguaje centrado en la muestra es la que presenta mayor cantidad de actividades en ambos programas.

En el caso del contexto, ambos programas de estudio ( $7^{\circ}$ y $8^{\circ}$ básico) evidencian que gran parte de las actividades propuestas presentan un contexto basado en experiencias de aprendizaje, aspecto que es coincidente con la escasa presencia de componentes de inferencia informal.

El incremento (en cantidad) presente en los programas vigentes no se ve reflejado en la estructura y objetivos de las actividades, puesto que en muy pocos casos hay evidencia de la integración de las componentes de inferencia informal y contexto para desarrollar inferencias.

\section{CONCLUSIONES Y PROPUESTAS}

A partir del análisis llevado a cabo se puede apreciar que la mayoría de las actividades que presentan los programas de estudio no muestran una integración de las componentes de inferencia informal. Las componentes que se presentan con mayor frecuencia son uso de datos como evidencia y posibilidad de generalización, en su mayoría integradas entre sí. Sin embargo, la mayor parte presenta un lenguaje centrado en la muestra. Este tipo de actividades promueve el análisis descriptivo de los datos de una muestra sin considerar que uno de los objetivos principales de las muestras estadísticas es proporcionar información sobre la población (Makar y Rubin, 2009). Es decir, una muestra de datos provee un espacio para inferir una característica de interés de la población, en cambio, el análisis de datos poblacionales tiene fines descriptivos (Pfannkuch y Wild, 2015). En este sentido, las actividades que proponen los programas de estudio Mineduc (2016a, 2016b) muestran contradicciones con los objetivos que se proponen en la base curricular de Matemática (Mineduc, 2015, pp. 105-115), pero estos mismos objetivos están en la misma sintonía con los lineamientos descritos en NCTM (2000) y GAISE (2005, 2016) para el eje de Estadística y Probabilidad. 
Otro de los resultados encontrados es la ambigüedad y descontextualización que presentan algunas de las actividades. Estas no especifican si los datos son parte de una muestra o una población ni tampoco (al menos de forma simple) la forma de selección de la muestra, generando sesgo en el desarrollo de inferencias o descripciones.

Sobre el contexto presentado en las actividades se visualizó que muchas promueven un contexto basado en experiencias de aprendizaje, haciendo énfasis en el cálculo de estadísticos, cálculo de porcentajes, construcción de gráficas alternativas, preguntas descriptivas etc. sin hacer hincapié que las medidas de centro, dispersión o posición de una muestra son estimadores de las medidas de la población. Esto no apoya el desarrollo de inferencias, pues este tipo de contexto promueve aspectos procedimentales en contraste con la profundización conceptual. El aprendizaje de la estadística bajo el uso de contexto en datos permite introducir a estudiantes en la práctica estadística, considerando patrones bajo la situación real y estableciendo interpretaciones adecuadas; no obstante, un exceso de actividades bajo este contexto puede interferir en el proceso de razonamiento inferencial informal. Por ello, como lo plantean Schwartz y Goldman (1996), se debe considerar el diseño de contextos variados de actividades en el proceso de aprendizaje. De acuerdo con Pfannkuch (2011), las actividades con un contexto basado en datos se deben mostrar equilibradas con aquellas del contexto basado en experiencias aprendizaje, pues ambas influyen en los procesos de razonamiento inferencia informal.

Las actividades incluidas en los programas de estudio vigentes de $7^{\circ}$ y $8^{\circ}$ básico (Mineduc (2016a; 2016b), si bien es cierto que muestran un incremento en relación a la cantidad de actividades con componentes de inferencia informal y/o de contexto, no es suficiente, dado que ambas propuestas buscan una integración de las componentes de inferencia informal y una mayor cantidad de actividades que permita a los estudiantes la manipulación con datos situados en su propia realidad. Los resultados encontrados evidencian la existencia de una brecha que se debe acortar sobre lo que proponen los objetivos curriculares y las actividades que se presenten en los programas de estudio vigentes, pues como se mencionó, este recurso es la base para el diseño de la enseñanza por los profesores. Así mismo, la intencionalidad debe ser coherente con base en las propuestas para la enseñanza de la estadística. Si bien es cierto que van emergiendo luces sobre la inclusión de aspectos que son relevantes para razonar y pensar estadísticamente, es deseable que esta brecha disminuya, de lo contrario el foco de la estadística se pierde, se potencia el trabajo mecanicista y rutinario que se refleja en las actividades y se incrementan las dificultades para profesores (Estrella, Olfos y Mena-Lorca, 2015; Vásquez y Alsina, 2014; Vásquez, Pincheira, Piñeiro y Diaz-Levicoy, 2019).

Este trabajo tiene la intención de profundizar en el análisis curricular en el área de estadística y brindar a los profesores una herramienta de trabajo que se relaciona 
directamente con su práctica de enseñanza. Con esto los profesores podrían rediseñar o enriquecer las actividades que se proponen tanto en programas de estudio y libros de texto focalizando la enseñanza en los supuestos que subyacen al análisis de datos, la integración del contexto y el desarrollo de inferencias informales sobre características de interés de una población, promoviendo la importancia de los aspectos conceptuales de la estadística.

\section{REFERENCIAS}

Aridor, K., y Ben-Zvi, D. (2018). Statistical modeling to promote students' aggregate reasoning with sample and sampling. $Z D M, 50(7), 1165-1181$.

Batanero, C. (2002). Los retos de la cultura estadística. Conferencia inaugural de Jornadas Interamericanas de Enseñanza de la Estadística. Buenos Aires, Argentina. Recuperado de: http://www.ugr.es/ batanero/ ARTICULOS/CULTURA.pdf.

Batanero, C., Gea, M., Arteaga, P., y Contreras, J. M. (2014). La estadística en la educación obligatoria: análisis del currículo español. Revista DigitalMatemática, Educación e Internet, 14(2). Recuperado de: http://www. tec-digital.itcr.ac.cr/revistamatematica/.

Ben-Zvi, D., y Garfield, J. B. (2004). Statistical literacy, reasoning and thinking: Goals, definitions, and challenges. En D. Ben-Zvi y J. B. Garfield (eds.), The challenge of developing statistical literacy, reasoning, and thinking (pp. 3-16). Kluwer Publishers.

Brown, M. W. (2009). The teacher-tool relationship: Theorizing the design and use of curriculum materials. En J. T. Remillard, B. A. Herbel-Eisenmann, y G. M. Lloyd (eds.), Mathematics teachers at work: Connecting curriculum materials and classroom instruction (pp. 17-36). Nueva York: Routledge.

Burrill, G., y Biehler, R. (2011). Fundamental statistical ideas in the school curriculum and in training teachers. En C. Batanero, G. Burrill, y C. Reading (eds.), Teaching statistics in school mathematics. Challenges for teaching and teacher education - A joint ICMI/LASE study (pp. 57-69). Dordrecht: Springer.

Cobo, B. (2003). Significado de las medidas de posición central para los estudiantes de secundaria [tesis doctoral]. Universidad de Granada.

Davis, E. A., y Krajcik, J. S. (2005). Designing educative curriculum materials to promote teacher learning. Educational Researcher, 34(3), 3-14.
Del Pino, G., y Estrella, S. (2012). Educación estadística: relaciones con la matemática. Pensamiento Educativo, 49(1), 53-64.

Díaz-Levicoy, D., Batanero, C., Arteaga, P., y Gea, M. (2015). Análisis de gráficos estadísticos en libros de texto de educación primaria española. Unión, 44, 90-112.

Doerr, H. M., delMas, R., y Makar, K. (2017). A modeling approach to the development of students' informal inferential reasoning. Statistics Education Research Journal, 16(2), 86-115.

Erickson, T. (2006). Using simulation to learn about inference. En A. Rossman y B. Chance (eds.), Working cooperatively in statistics education. Proceedings of the Seventh International Conference on Teaching Statistics, Salvador, Brazil. [CDROM]. Voorburg, Países Bajos: International Statistical Institute. Recuperado de: http://www. stat.auckland.ac.nz/ iase/publications/17/7G2_ ERIC.pdf.

Estrella, S., Olfos, R., y Mena-Lorca, A. (2015). El conocimiento pedagógico del contenido de estadística en profesores de primaria. Revista Educacao e Pesquisa, 41(2), 477-493.

Franklin, C., Kader, G., Mewborn, D. S., Moreno, J., Peck, R., Perry, M., y Scheaffer, R. (2005). A curriculum framework for K-12 statistics education. GAISE Report. American Statistical Association. Recuperado de: http://www.amstat.org/education/gaise/.

GAISE (2016). Guidelines for Assessment and Instruction in Statistics Education: College report. American Statistical Association. Recuperado de: http://www.amstat.org/ ASA/Education/Undergraduate-Educators.

Gal, I. (2019). Understanding statistical literacy: About knowledge of contexts and models. En J. M. Contreras, M. M. Gea, M. M. López-Martín y E. MolinaPortillo (eds.), Actas del Tercer Congreso International 
Virtual de Educación Estadística. Recuperado de: www. ugr.es/local/fqm126/ civeest.html.

García-Ríos, V. (2013). Inferencias estadísticas informales en estudiantes mexicanos. En J. M. Contreras, G. R. Cañadas, M. M. Gea y P. Arteaga (eds.), Actas de las Jornadas Virtuales en Didáctica de la Estadística, Probabilidad y Combinatoria, 1, 343-357.

García-Ríos, V. N., y Sánchez, E. (2015). Dificultades en el razonamiento inferencial intuitivo. En J. M. Contreras, C. Batanero, J. D. Godino, G. R. Cañadas, P. Arteaga, E. Molina, M. M. Gea y M. M. López (eds.), Didáctica de la estadística, probabilidad y combinatoria 2 (pp. 207-214). Granada, España: Sociedad Española de Investigación en Educación Matemática.

Garfield, J., y Ben-Zvi, D. (2008). Developing students'statistical reasoning: Connecting research and teaching practice. Dordrecht, Países Bajos: Springer.

Krippendorff, K. (1997). Metodología de análisis de contenido. Teoría y práctica. Barcelona: Paidós Comunicación.

Konold, C., Higgins, T., Russell, S.-J., y Khalil, K. (2015). Data seen through different lenses. Educational Studies in Mathematics, 88(3), 305-325.

Makar, K., y Rubin, A. (2009). A framework for thinking about informal statistical inference. Statistics Education Research Journal, 8(1), 82-105. Recuperado de: http://www.stat.auckland.ac.nz/ iase/serj/ SERJ8(1)_Makar_Rubin.pdf.

Makar, K., Bakker, A., y Ben-Zvi, D. (2011). The reasoning behind informal statistical inference. Mathematical Thinking and Learning, 13, 152-173.

Maldonado, J., y Ojeda, A. M. (2009). Ideas fundamentales de estadística en educación primaria: una perspectiva epistemológica. Revista Premisa, 11(43), 3-10.

MEC (2007). ORDEN ECI/2211/2007, del 20 de julio, por la que se establece el curriculo y regula la ordenación de la educación primaria. Madrid: Autor.

Mineduc (2000). Programa de estudio Séptimo año Básico / Nivel Básico 5 Educación Básica. Unidad de Currículum y Evaluación. Santiago, Chile.

Mineduc (2002). Programa de estudio Octavo año Básico / Nivel Básico 6 Educación Básica. Unidad de Currículum y Evaluación. Santiago, Chile.

Mineduc (actualización 2009). Marco curricular de objetivos fundamentales y contenidos mínimos obligatorios de la Educación Básica y Media. Ministerio de Educación. Chile.
Mineduc (2015). Bases curriculares de $7^{\circ}$ básico a $2^{\circ}$ medio. Matemática. Santiago: Ministerio de Educación.

Mineduc (2016a). Matemática, Programa de estudio Séptimo básico. Unidad de Currículum y Evaluación. Santiago, Chile.

Mineduc (2016b). Matemática, Programa de estudio Octavo básico. Unidad de Currículum y Evaluación. Santiago, Chile.

Morales, R., y Ruiz, K. (2013). Comparación entre los contenidos del currículo chileno y español en el área de estadística y probabilidad. En J. M. Contreras, G. R. Cañadas, M. M. Gea y P. Arteaga (eds.), Actas de las Jornadas Virtuales en Didáctica de la Estadística, Probabilidad y Combinatoria (pp. 137-142). Granada, Departamento de Didáctica de la Matemática de la Universidad de Granada.

NCTM (2000). Principles and standards for school mathematics. Reston, VA.: Autor.

López-Mojica, J. M. (2013). Pensamiento probabilístico y esquemas compensatorios en la educación especial [tesis de doctorado no publicada]. Centro de Investigación y de Estudios Avanzados del IPN, México.

López-Mojica, J. M., Ojeda, A. M., y Salcedo, J. (2018). Ideas fundamentales de estocásticos en libros de texto de educación primaria: una alternativa de enseñanza. IE Revista de Investigación Educativa de la REDIECH, 9(17), 87-102.

Pérez-Serrano, G. (1994). Investigación cualitativa: retos e interrogantes. Madrid: La Muralla.

Pérez, Y., Ruiz, B., y Hugues, E. (2019). Análisis de actividades estadísticas en libros de textos de nivel básico y medio superior en México. Revista Digital: Matemática, Educación e Internet, 19(2).

Pfannkuch, M. (2005). Probability and statistical inference: How can teachers enable learners to make the connection? En G. A. Jones (ed.), Exploring probability in school: Challenges for teaching and learning (pp. 267-294). Nueva York: Springer.

Pfannkuch, M. (2011). The role of context in developing informal statistical inferential reasoning: A classroom study. Mathematical Thinking and Learning, 13(1-2), 27-46.

Pfannkuch, M., y Wild, C. (2004). Towards an understanding of statistical thinking. En D. Ben-Zvi y J. Garfield (eds.), The challenge of developing statistical literacy, 
reasoning, and thinking (pp. 17-47). Dordrecht, Países Bajos: Kluwer.

Pfannkuch, M., y Wild, Ch. J. (2015). Laying foundations for statistical inference. En Cho, S. (ed.), Selected regular lectures from the 12th International Congress on Mathematical Education (pp. 653-666). Cham: Springer.

Remillard, J. T. (2000). Can curriculum materials support teacher's learning? Elementary School Journal, 100, 331-350.

Remillard, J. T., y Bryans, M. B. (2004). Teachers' orientations toward mathematics curriculum materials: Implications for teacher learning. Journal for Research in Mathematics Education, 35(5), 352-388.

Schwartz, D., y Goldman, S. (1996). Why people are not like marbles in an urn: An effect of context on statistical reasoning. Applied Cognitive Psychology, 10, S99-S112.

Vásquez, C. (2018). Surgimiento del lenguaje probabilístico en el aula de educación primaria. REnCiMa, 9(2), 374-389.
Vásquez, C., y Alsina, Á. (2014). Enseñanza de la probabilidad en educación primaria. Un desafío para la formación inicial y continua del profesorado. Números, 85, 5-23.

Vásquez, C., Pincheira, N., Piñeiro, J. L., y Diaz-Levicoy, D. (2019). ¿Cómo se promueve el aprendizaje de la estadística y la probabilidad? Un análisis desde los libros de texto para la Educación Primaria. BOLEMA. Boletim de Educação Matemática, 33(65), 1133-1154.

Watson, J. M. (2006). Statistical literacy at school: Growth and goals. Mahwah, NJ: Lawrence Erlbaum.

Wild, C. J., y Pfannkuch, M. (1999). Statistical thinking in empirical enquiry. International Statistical Review, 67(3), 223-248.

Zieffler, A., Garfield, J., delMas, R., y Reading, C. (2008). A framework to support research on informal inferential reasoning. Statistics Education Research Journal, 7(2), 40-58.

Cómo citar este artículo:

Sánchez Acevedo, N. A. y Ruiz Hernández, B. R. (2020). Análisis de las actividades propuestas en dos programas de estudio chilenos en el eje de Estadística y Probabilidad. IE Revista de Investigación Educativa de la REDIECH, 11, e-776. doi: http://dx.doi.org/10.33010/ie_rie_rediech.v11i0.776. 